\title{
Idiopathic REM Sleep Behavior Disorder: Implications for the Pathogenesis of Lewy Body Diseases
}

\author{
Tomoyuki Miyamoto, Masayuki Miyamoto, Masaoki Iwanami, and Koichi Hirata \\ Department of Neurology, Center of Sleep Medicine, School of Medicine, Dokkyo Medical University, 880 Kitakobayashi, Mibu, \\ Shimotsuga, Tochigi 321-0293, Japan \\ Correspondence should be addressed to Tomoyuki Miyamoto, miyatomo@dokkyomed.ac.jp
}

Received 11 October 2010; Accepted 15 February 2011

Academic Editor: Irena Rektorova

Copyright ( $\odot 2011$ Tomoyuki Miyamoto et al. This is an open access article distributed under the Creative Commons Attribution License, which permits unrestricted use, distribution, and reproduction in any medium, provided the original work is properly cited.

\begin{abstract}
Objectives. Both results of the odor identification and cardiac ${ }^{123}$ I-metaiodobenzylguanidine accumulation have been investigated for their potential to enhance the detection of pathogenesis resembling that of Lewy body-related $\alpha$-synucleinopathies in patients clinically diagnosed as having idiopathic REM sleep behavior disorder. Methods. We performed both the Odor Stick Identification Test for Japanese and ${ }^{123}$ I-metaiodobenzylguanidine scintigraphy in 30 patients with idiopathic REM sleep behavior disorder, 38 patients with Parkinson's disease, and 20 control subjects. Results. In idiopathic REM sleep behavior disorder, reduced odor identification score and an early or delayed heart to mediastinum ratio on ${ }^{123}$ I-metaiodobenzylguanidine were almost as severe as in Parkinson's disease patients. Delayed cardiac ${ }^{123}$ I-metaiodobenzylguanidine uptake was even more severe in the idiopathic REM sleep behavior disorder group than in the Parkinson's disease group. Conclusions. Reduced cardiac ${ }^{123}$ I-metaiodobenzylguanidine uptake, which is independent of parkinsonism, may be more closely associated with idiopathic REM sleep behavior disorder than olfactory impairment.
\end{abstract}

\section{Introduction}

REM sleep behavior disorder (RBD) is characterized by dream-enacting behaviors and unpleasant dreams and presents a risk for self-injury and harm to others due to abnormal REM sleep during which control of muscle tonus is lacking (REM sleep without atonia) $[1,2]$. RBD is a heterogeneous disease entity consisting of a variety of manifestations [1]. Idiopathic RBD (iRBD), which develops in middle age or later and progresses chronically, in particular is a common clinical manifestation of Lewy body-related syndrome and is regarded as a clinical entity from the pathological aspect. For example, it has been elucidated that iRBD is often accompanied by soft motor signs, olfactory and color identification deficits, decreased cardiovascular and respiratory changes between REM and NREM sleep, reduced cardiac ${ }^{123}$ I-metaiodobenzylguanidine ( $\left.{ }^{123} \mathrm{I}-\mathrm{MIBG}\right)$ uptake, impairment of visual memory and visuospatial construction on neuropsychological testing, EEG slowing during wakefulness or sleep, and decreased strial dopaminergic innervations, and reduced presynaptic strial dopamine transporter binding on SPECT or positron emission topography (PET) scans, which are considered as nonmotor symptoms of Parkinson's disease (PD) [3-5]. Furthermore, despite the limited number of pathological reports on $\mathrm{iRBD}$, the characteristics of $\mathrm{iRBD}$ have been supported to have a close relationship with Lewy body pathology [6]. Therefore, additional clinical features that could distinguish iRBD with Lewy body-related $\alpha$ synucleinopathies from iRBD from other causes would be helpful in clinical practice. Recently, an association between loss of olfactory function and loss of cardiac noradrenergic innervation in PD has been noted [7]. Moreover, both loss of sense of smell and cardiac sympathetic denervation can precede the onset of motor symptoms, suggesting that the combination might provide a biomarker for the risk of Lewy body disease [8].

In this study, by the combination of tests to detect odor identification [9] and to determine cardiac ${ }^{123}$ I-MIBG cardiac uptake $[4,5]$ that were selected from among various tests to examine nonmotor symptoms of PD, we investigated 
TABLE 1: Clinical characteristics of patients in the iRBD, PD, and control groups.

\begin{tabular}{|c|c|c|c|c|}
\hline & iRBD & PD & Controls & $P$ value \\
\hline Number of patients, $(n)$ & 30 & 38 & 20 & - \\
\hline Age, years & $65.8 \pm 9.1$ & $65.4 \pm 9.2$ & $62.3 \pm 6.8$ & $0.210^{\mathrm{a}}$ \\
\hline Sex (male, \%) & 83.3 & 63.2 & 90.0 & $0.102^{\mathrm{c}}$ \\
\hline Disease duration, years & $5.7 \pm 8.8$ & $4.8 \pm 4.8$ & N/A & $0.599^{\mathrm{b}}$ \\
\hline MMSE (>24) & $27.7 \pm 2.3$ & $27.9 \pm 1.9$ & $28.8 \pm 1.4$ & $0.096^{\mathrm{a}}$ \\
\hline Hoehn and Yahr stage & N/A & $2.4 \pm 0.8$ & N/A & N/A \\
\hline UPDRS motor subset, score & $0.6 \pm 1.0$ & $14.4 \pm 7.8$ & N/A & $0.000^{\mathrm{b}}$ \\
\hline LEU, mg/day & N/A & $238.6 \pm 261.9$ & N/A & N/A \\
\hline Smoker, $(\%)$ & 50.0 & 39.5 & 5.0 & $0.464^{\mathrm{c}}$ \\
\hline OSIT-J, score & $5.2 \pm 3.0$ & $4.4 \pm 2.4$ & $10.3 \pm 1.3$ & $0.000^{\mathrm{a}}$ \\
\hline \multicolumn{5}{|l|}{${ }^{123} \mathrm{I}-\mathrm{MIBG}$} \\
\hline Early $\mathrm{H} / \mathrm{M}$ ratio & $1.85 \pm 0.33$ & $2.14 \pm 0.52$ & $2.74 \pm 0.33$ & $0.000^{\mathrm{a}}$ \\
\hline Delayed H/M ratio & $1.46 \pm 0.29$ & $1.83 \pm 0.60$ & $2.82 \pm 0.47$ & $0.000^{\mathrm{a}}$ \\
\hline
\end{tabular}

Data are mean \pm SD.

PD: Parkinson's disease, iRBD: idiopathic REM sleep behavior disorder, OSAS: obstructive sleep apnea syndrome, MMSE: Mini-Mental State Examination, UPDRS: Unified Parkinson's Disease Rating Scale, LEU: daily levodopa-equivalent unit, OSIT-J: Odor Stick Identification Test for Japanese, H/M: heart-tomediastinum, and ${ }^{123}$ I-MIBG: ${ }^{123}$ I-metaiodobenzylguanidine.

a $P$ value was determined by one-way ANOVA.

${ }^{\mathrm{b}} P$ value was determined by Mann-Whitney $\mathrm{U}$ test.

${ }^{\mathrm{c}}$ Chi-square test for categorical: PD versus iRBD.

if there was an association between olfaction and cardiac ${ }^{123}$ I-MIBG uptake in patients with $\mathrm{iRBD}$ and PD to evaluate the correlation between results of these two tests and to determine which would more enhance the detection of pathogenesis resembling that of Lewy body-related $\alpha$ synucleinopathies in patients clinically diagnosed as having iRBD.

\section{Methods}

2.1. Patient Selection. This study was performed in accordance with the Declaration of Helsinki. Procedures were approved by the Ethics Review Committee of Dokkyo Medical University, and informed consent was obtained from each subject. Subjects were 82 patients matched according to age group: 30 had iRBD (iRBD group; $66.3 \pm 5.7$ years; 25 males, 5 females), 38 had PD (PD group; $65.4 \pm 9.2$ years; 24 males, 14 females), and 20 had obstructive sleep apnea (OSA) without RBD (control group; $62.3 \pm 6.8$ years; 18 males, 2 females; pretreatment $\mathrm{AHI}=38.5 \pm 31.3$ events/h) (Table 1$)$. $\mathrm{RBD}$ and OSAS were defined according to the International Classification of Sleep Disorders, second edition [2]. Exclusion criteria were an abnormal neurologic examination in subjects with iRBD and OSAS. PD patients fulfilled the United Kingdom Parkinson's Disease Society Brain Bank criteria for idiopathic PD [10]. Parkinsonism was assessed and classified according to the Hoehn and Yahr stage [11] and the motor subset of Unified Parkinson's Disease Rating Scale (UPDRS-III), which was administered to the PD and iRBD groups [12]. The mean UPDRS-III score was $14.4 \pm 7.8$ points for PD patients and $0.6 \pm 1.0$ points for the iRBD subjects. There were two phenotypes of PD among the PD group: tremor-dominant and postural instability gait difficulty(PIGD-) dominant based on UPDRS components. The mean
PIGD score was defined as the sum of an individual's baseline falling, freezing, walking, gait, and postural stability scores divided by 5 . Patients were categorized as having tremordominant PD if the ratio of the mean tremor score to the mean PIGD score was 1.5 or higher and as having PIGDdominant PD if that ratio was 1.00 or lower [13]. According to that categorization, 13 patients in the PD group were placed in the tremor-dominant subgroup and 21 in PIGDdominant subgroup. For this particular study, all patients with Mini-Mental State Examination (MMSE) scores <24 were excluded [14].

\subsection{Polysomnographic Evaluation and Definition of $i R B D$.} Polysomnographic (PSG) monitoring included electroencephalography (C3, C4, O1, and O2), electrooculography, chin muscle electromyography (EMG), electrocardiography, detection of airflow by thermistor, plethysmography for ribcage and abdominal wall motion, oximetry for measurement of arterial oxyhemoglobin saturation, detection of changes in sleeping position, and bilateral EMG of the tibialis anterior muscles. PSG monitoring was performed for at least $8 \mathrm{~h}$. Sleep stages were manually scored according to criteria of Rechtschaffen and Kales [15]. Apnea was defined as the absence of breathing for more than $10 \mathrm{~s}$. Hypopnea was defined as a reduction of more than $50 \%$ in breathing; when the reduction in breathing was less than 50\%, more than 3\% oxygen desaturation or arousal for more than $10 \mathrm{~s}$ was defined as hypopnea. The apnea-hypopnea index was calculated as the average of the total number of apnea and hypopnea episodes experienced per hour of sleep. For the iRBD group, the presence of REM sleep without atonia was based on EMG findings of excessive sustained or intermittent elevation of submental EMG tone or excessive phasic submental or lower limb EMG twitching according to the ICSD-2ed [2]. 
TABLE 2: Multiple regression analysis of OSIT-J related parameters for Parkinson's disease $(N=38)$ and iRBD $(N=30)$.

\begin{tabular}{|c|c|c|c|c|}
\hline & \multirow{2}{*}{$\begin{array}{c}\mathrm{PD} \\
\beta^{\mathrm{a}}\end{array}$} & \multicolumn{3}{|c|}{ iRBD } \\
\hline & & $P$ value & $\beta^{\mathrm{a}}$ & $P$ value \\
\hline$\overline{\text { Age }}$ & -0.348 & 0.037 & 0.016 & 0.936 \\
\hline Sex & 0.404 & 0.033 & 0.080 & 0.701 \\
\hline Disease duration, month & 0.147 & 0.591 & -0.314 & 0.377 \\
\hline MMSE (>24) & 0.189 & 0.230 & 0.184 & 0.377 \\
\hline Hoehn and Yahr stage & -0.013 & 0.940 & N/A & N/A \\
\hline UPDRS motor subset & 0.079 & 0.674 & -0.153 & 0.517 \\
\hline Motor phenotype & -0.239 & 0.177 & N/A & N/A \\
\hline LEU & 0.074 & 0.969 & N/A & N/A \\
\hline Smoking atatus & 0.007 & 0.798 & -0.040 & 0.834 \\
\hline Delayed $\mathrm{H} / \mathrm{M}$ ratio $\left({ }^{123} \mathrm{I}-\mathrm{MIBG}\right)$ & 0.399 & 0.015 & -0.274 & 0.183 \\
\hline $\mathrm{R}^{2^{\mathrm{b}}}$ & 0.493 & & 0.184 & \\
\hline
\end{tabular}

iRBD: idiopathic REM sleep behavior disorder, PD: Parkinson's disease, OSIT-J: Odor Stick Identification Test for Japanese, MMSE: Mini-Mental State Examination, UPDRS: Unified Parkinson's Disease Rating Scale, LEU: daily levodopa-equivalent unit, H/M: heart-to-mediastinum, and ${ }^{123}$ I-MIBG: ${ }^{123}$ Imetaiodobenzylguanidine.

${ }^{a}$ Standardized regression coefficients.

${ }^{\mathrm{b}}$ Coefficient of determination.

Bold values indicate statistically significant values. N/A: not aplicable.

2.3. Cardiac ${ }^{123}$ I-MIBG Scintigraphy. ${ }^{123} \mathrm{I}-\mathrm{MIBG}$ planar images of the chest were obtained using a triple-headed gamma camera (GCA-9300A-HG, Toshiba Co, Tokyo, Japan). ${ }^{123} \mathrm{I}-$ MIBG (Fujifilm RI Pharma Co., Tokyo, Japan) accumulations were recorded at the early $(15 \mathrm{~min})$ and delayed phases $(4 \mathrm{~h})$. The heart-to-mediastum $(\mathrm{H} / \mathrm{M})$ ratio was calculated by dividing the count density of the left ventricular region of interest (ROI) by that of the mediastinal ROI [16-18]. None of the subjects took medications that could influence the ${ }^{123}$ I-MIBG examination, and none had a history of cardiac disease.

2.4. Odor Stick Identification Test for Japanese (OSIT-J). The Odor Stick Identification Test for Japanese (OSIT-J) (Daiichi Yakuhin, Co., Ltd., Tokyo, Japan) is composed of 12 different odorants familiar to the Japanese population [9]. All subjects were free from other conditions that can affect olfactory function such as usage of certain medications, chronic nasal infection, chronic sinonasal disease, head trauma, and abuse of drugs or alcohol by medical history. Subjects with an infection of the upper airways at the time of the investigation were also not allowed to participate. OSIT-J was performed as previously described. In this study, functional hyposmia was defined by an OSIT-J score $\leq 8$ [9].

2.5. Statistical Analysis. Values are expressed as mean $\pm \mathrm{SD}$. Age, MMSE, OSIT-J scores and degrees of accumulation based on the $\mathrm{H} / \mathrm{M}$ ratio (early and delayed phase) in cardiac muscle obtained were compared among the three groups by the one-way analysis of variance (ANOVA) followed by posthoc Bonferroni correction. The Mann-Whitney U-test was applied for statistical comparisons of disease duration and scores of the motor subsets of the UPDRS between the iRBD and PD groups. Group comparisons of the frequency of genders and smokers were performed by the Chi-square test for categorical variables. The correlations of OSIT-J and early or delayed ${ }^{123}$ I-MIBG uptake were assessed by the Spearman correlation coefficient for iRBD and PD. To explore the most influential factor on the OSIT-J score, we included age, sex, disease duration, MMSE, Hoehn and Yahr stage, UPDRS motor subsets, motor phenotypes, levodopa-equivalent unit (LEU), smoking status, and delayed ${ }^{123}$ I-MIBG uptake in a multiple regression analysis for PD. Receiver operating characteristic (ROC) curves were analyzed. The significance level was set at $P<.05$. Statistical analyses were performed with the Statistical Package for Social Science Software (Graphpad Prism, San Diego, CA and SPSS II Windows Ver 11.0, Japan).

\section{Results}

A total of $30 \mathrm{iRBD}$ patients (mean symptom duration: $5.7 \pm$ 8.8), 38 PD patients, and 20 control subjects with a similar age distribution were evaluated. Demographic information is presented in Table 1 . Interval between ${ }^{123} \mathrm{I}-\mathrm{MIBG}$ examinations and OSIT-J was a mean of 72 days.

Cardiac ${ }^{123}$ I-MIBG accumulation based on H/M (early phase, delayed phase) was significantly decreased in the iRBD group $(1.85 \pm 0.33,1.46 \pm 0.29)$ and the PD group $(2.14 \pm 0.52$, $1.83 \pm 0.60)$ compared with the control group $(2.74 \pm 0.33$, $2.82 \pm 0.47)(P<.000)$. OSIT-J scores were significantly lower in the iRBD group $(5.2 \pm 3.0)$ and the PD group $(4.4 \pm 2.4)$ than in the control group $(10.3 \pm 1.3)(P<.000)$ (Table 1$)$. As shown in Table 2, results of multiple regression analysis of OSIT-J-related parameters in patients with PD, which included age, sex, disease duration, MMSE, Hoehn and Yahr stage, UPDRS motor subset, motor phenotype, LEU, smoking status, and delayed H/M ratio, showed that age, sex, and delayed $\mathrm{H} / \mathrm{M}$ ratio were the significant factors in the model for the OSIT-J score. There was a significant positive correlation between the OSIT-J score and delayed H/M ratio 


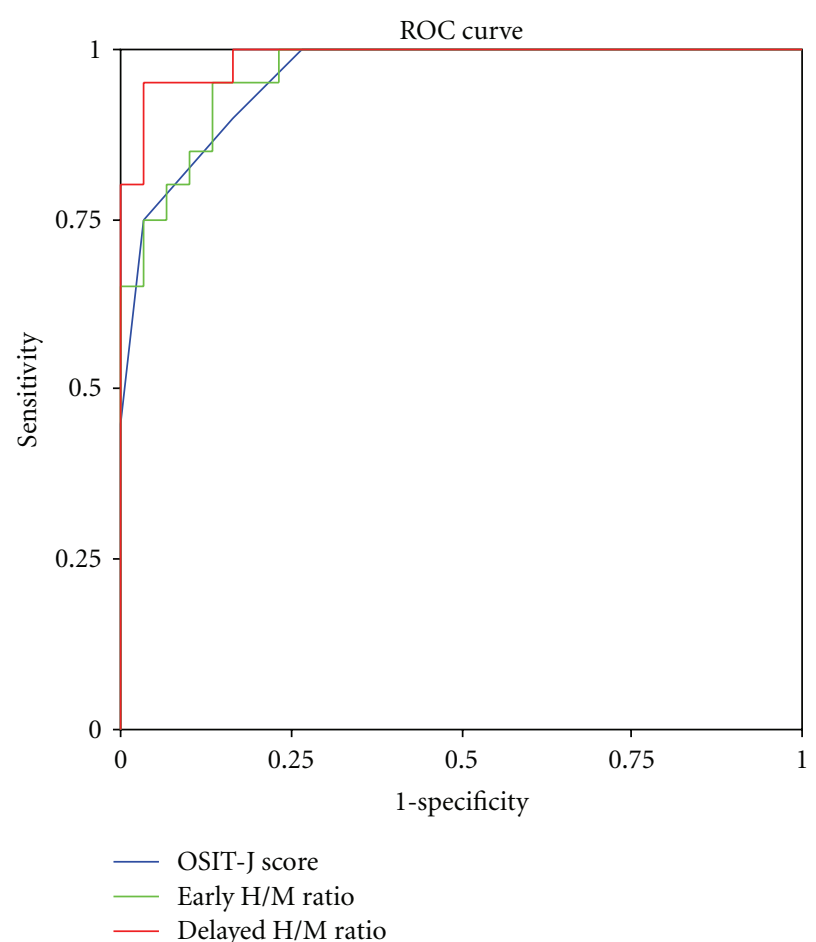

(a)

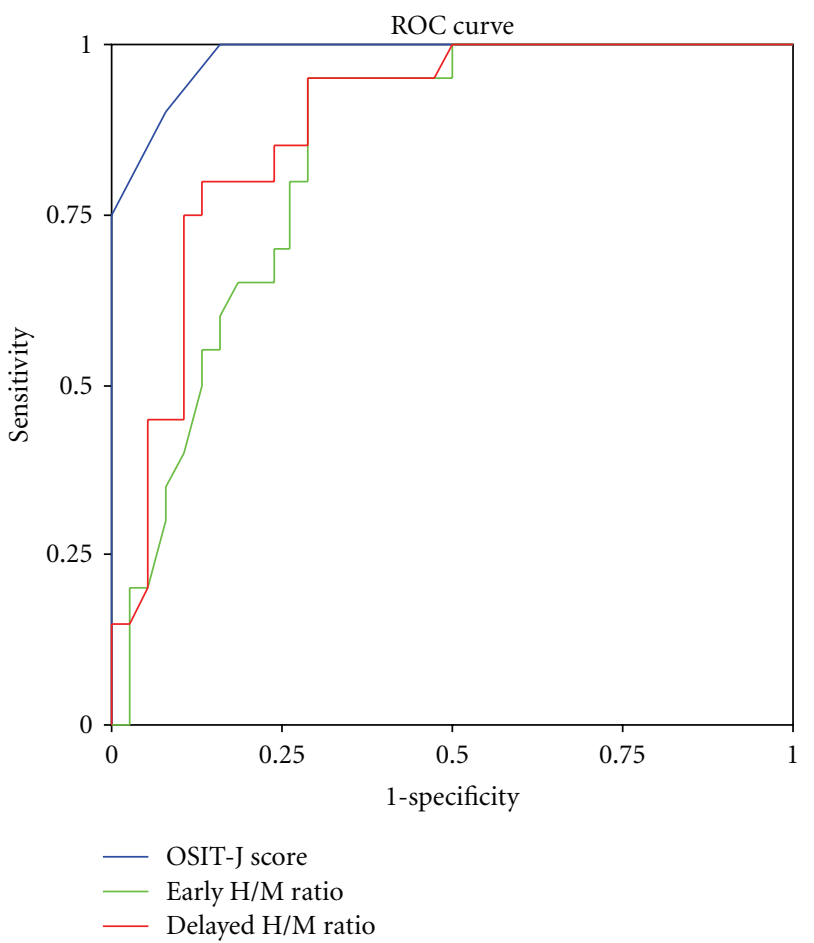

(b)

FIGURE 1: (a) ROC analysis of the early or delayed H/M ratio and odor identification showed an area under the curve of $0.96(95 \% \mathrm{CI}=0.92-$ $1.00, P=.000), 0.99(95 \% \mathrm{CI}=0.96-1.00, P=.000)$, and $0.96(95 \% \mathrm{CI}=0.91-1.00, P=.000)$ for iRBD. (b) ROC analysis of the early or delayed $\mathrm{H} / \mathrm{M}$ ratio and odor identification showed an area under the curve of $0.84(95 \% \mathrm{CI}=0.74-0.94, P=.000), 0.88(95 \% \mathrm{CI}=0.79-$ $0.97, P=.000)$, and $0.98(95 \% \mathrm{CI}=0.96-1.00, P=.000)$ for PD.

TABLE 3: Sensitivity and specificity of OSIT-J and $\operatorname{cardiac}^{123}$ I-MIBG scintigraphy.

\begin{tabular}{lccc}
\hline & OSIT-J score & Early H/M ratio & $\begin{array}{c}\text { Delayed } \\
\text { H/M ratio }\end{array}$ \\
\hline $\begin{array}{l}\text { iRBD patients } \\
(n=30)\end{array}$ & & & \\
$\quad$ Cut-off & 8.5 & 2.30 & 2.03 \\
$\quad$ Sensitivity & $90.0 \%$ & $95.0 \%$ & $95.0 \%$ \\
$\quad$ Specificity & $83.3 \%$ & $86.7 \%$ & $96.7 \%$ \\
PD patients & & & \\
$(n=38)$ & & & \\
$\quad$ Cut-off & 8.5 & 2.30 & 2.03 \\
$\quad$ Sensitivity & $90.0 \%$ & $95.0 \%$ & $95.0 \%$ \\
$\quad$ Specificity & $92.1 \%$ & $71.1 \%$ & $71.1 \%$ \\
\hline
\end{tabular}

iRBD: idiopathic REM sleep behavior disorder, PD: Parkinson's disease, OSIT-J: Odor Stick Identification Test for Japanese, ${ }^{123} \mathrm{I}-\mathrm{MIBG}:{ }^{123} \mathrm{I}-$ metaiodobenzylguanidine, H/M: heart-to-mediastinum.

( $r=0.337, P=.039$, Spearman's correlation). On the other hand, results of multiple regression analysis of OSITJ-related parameters in patients with iRBD, which included age, sex, disease duration, MMSE, UPDRS motor subset, smoking status, and delayed $\mathrm{H} / \mathrm{M}$ ratio, showed that there were no significant factors in the model for the OSIT-J score. Among the iRBD group, there was no significant correlation between the OSIT-J score and delayed H/M ratio $(r=$ $-0.195, P=.302$, Spearman's correlation) or the duration of RBD ( $r=-0.217, P=.249$, Spearman's correlation).

ROC analysis of the early or delayed $\mathrm{H} / \mathrm{M}$ ratio and odor identification showed an area under the curve of 0.96 (95\% CI $=0.92-1.00, P=.000), 0.99$ (95\% CI $=0.96-1.00$, $P=.000)$ and $0.96(95 \% \mathrm{CI}=0.91-1.00, P=.000)$ for $\mathrm{iRBD}$ (Figure $1(\mathrm{a})$ ) and of $0.84(95 \% \mathrm{CI}=0.74-0.94, P=.000)$, $0.88(95 \% \mathrm{CI}=0.79-0.97, P=.000)$ and $0.98(95 \% \mathrm{CI}=$ $0.96-1.00, P=.000$ ) for PD (Figure 1(b)).

For differentiating the iRBD patients from control subjects, the sensitivity and specificity of the OSIT-J score with a cut-off value at 8.5 were $90.0 \%$ and $83.3 \%$, respectively; those of the early $\mathrm{H} / \mathrm{M}$ ratio with a cut-off value at 2.30 were $95.0 \%$ and $86.7 \%$, respectively, and those of a delayed $\mathrm{H} / \mathrm{M}$ ratio with a cut-off value at 2.03 were $95.0 \%$ and $96.7 \%$, respectively (Table 3 ). For differentiating PD patients from control subjects, the sensitivity and specificity of the OSIT-J score with a cut-off value at 8.5 were $90.0 \%$ and $92.1 \%$, respectively; those of the early $\mathrm{H} / \mathrm{M}$ ratio with a cutoff value at 2.30 were $95.0 \%$ and $71.1 \%$, respectively; and those of the delayed $\mathrm{H} / \mathrm{M}$ ratio with a cut-off value at 2.03 were $95.0 \%$ and $71.1 \%$, respectively (Table 3 ). With optimal cut-off values set at 2.03 for the delayed $\mathrm{H} / \mathrm{M}$ ratio and at 8.5 for OSIT-J score for iRBD patients (Table 3, Figure 2), identification of iRBD was confirmed in 23 (76.7\%) patients in the iRBD group through abnormal results of both of these 


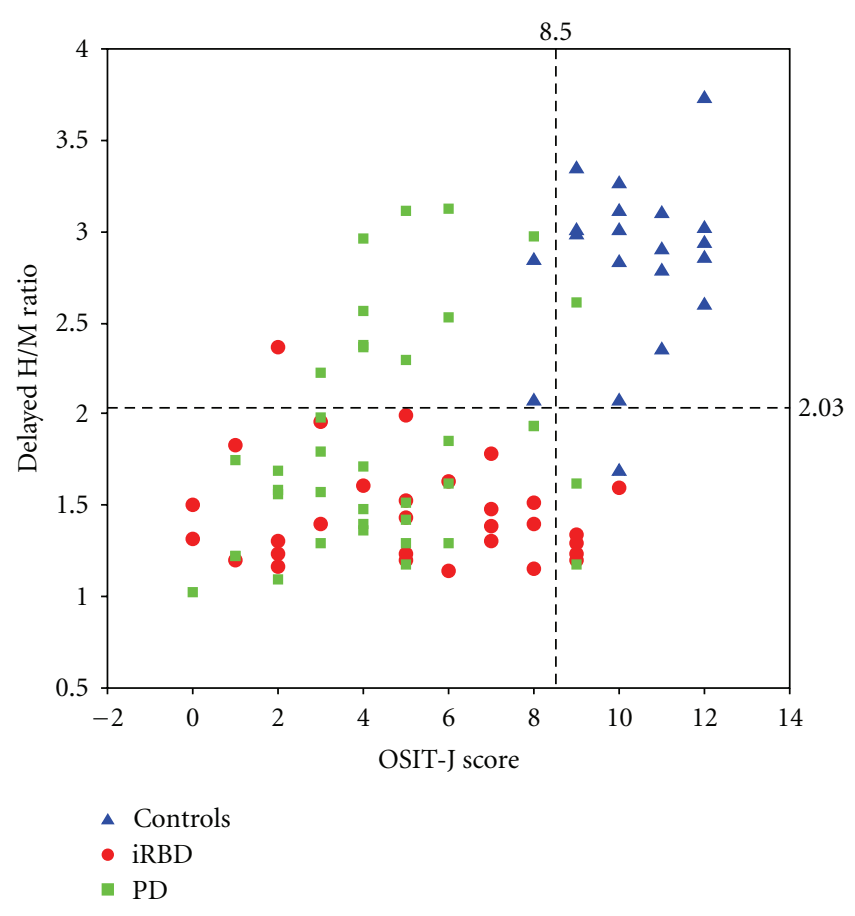

FIgURE 2: The relation between the OSIT-J score and the delayed $\mathrm{H} / \mathrm{M}$ ratio of ${ }^{123} \mathrm{I}-\mathrm{MIBG}$ uptake in patients with $\mathrm{iRBD}$ (the red circle), PD (the green square), and controls (the blue triangle). Broken line (vertical): cut-off value set at 8.5 by OSIT-J to distinguish between iRBD or PD and controls. Broken line (horizontal): cut-off value set at 2.03 by ${ }^{123}$ I-MIBG to distinguish between iRBD or PD and controls. OSIT-J: Odor Stick Identification Test for Japanese; H/M: heart-to-mediastinum; ${ }^{123} \mathrm{I}-\mathrm{MIBG}:{ }^{123} \mathrm{I}-$ metaiodobenzylguanidine; OSAS: obstructive sleep apnea syndrome; iRBD: idiopathic rapid eye movement behavior disorder; PD: Parkinson's disease.

tests. Optimal cut-off values were set at 2.03 for delayed H/M ratio and at 8.5 for OSIT-J score for PD patients (Table 3, Figure 2); as a result, 25 (65.8\%) were confirmed through abnormal results of both of these tests to have PD. However, results of both of these tests were normal in the control group.

\section{Discussion}

In this study, the olfactory dysfunction and reduced cardiac ${ }^{123}$ I-MIBG uptake that were found in most iRBD patients share similarities with those described in PD. Moreover, delayed cardiac ${ }^{123}$ I-MIBG uptake was more severe in the iRBD group than in the PD group (Figure 1(a)) while reduced odor identification was more severe in those with $\mathrm{PD}$ than with iRBD (Figure1(b)). Also, the degree of olfactory identification impairment correlated significantly with the degree of delayed cardiac ${ }^{123}$ I-MIBG uptake only in the PD group. Age, sex, and delayed cardiac ${ }^{123}$ I-MIBG uptake were significant factors with regard to PD patients in the model using the OSIT-J score. The degree of reduction of MIBG uptake does not match the degree of reduction in odor identification in iRBD patients but does match in PD patients. Results of a recent study indicated that the cardiac sympathetic nervous system might degenerate in parallel with the olfactory system in patients with early PD and that these two systems might degenerate at different rates of speed in advanced PD [16].

4.1. Cardiac Sympathetic Denervation Is Related to Lewy Body Pathology. There is evidence that ${ }^{123}$ I-MIBG cardiac uptake is markedly reduced in patients with Lewy body diseases such as PD, dementia with Lewy bodies (DLB), and pure autonomic failure (PAF) [17-19]. In was reported that cardiac ${ }^{123}$ I-MIBG imaging could distinguish between clinically diagnosed DLB and Alzheimer's disease (AD) with high levels of sensitivity and specificity [19]. Interestingly, pathological findings occur even in patients with DLB who have no parkinsonism. Since PAF patients do not have parkinsonism or decreased striatal dopaminergic innervation and since cardiac noradrenergic denervation occurs in both diseases, the pathogenetic mechanisms of cardiac noradrenergic denervation in Lewy body diseases differ from those producing parkinsonism and nigrostriatal dopaminergic denervation [8]. As pathological evidence [20-22], it has been proven that the Lewy body is present in cardiac sympathetic nerve postganglionic fibers and it has been suggested that Lewy body-related pathology potentially causes severe denervation and reduced ${ }^{123} \mathrm{I}-\mathrm{MIBG}$ uptake in the cardiac postganglionic sympathetic nerve. The reduction in ${ }^{123}$ I-MIBG uptake in sympathetic terminals was observed in cases with early-phase PD and incidental Lewy body disease (ILBD) irrespective of the presence or absence of remarkable autonomic nerve injury [17].

4.2. Olfactory Dysfunction Is Related to Lewy Body Pathology. Olfactory disturbance is present in most PD or PAF cases $[8,23,24]$. The deficit in olfaction in PD contrasts with previous reports of preserved or only mildly reduced olfaction in patients with atypical parkinsonism such as a tauopathy or multiple system atrophy $[25,26]$. In dementia patients, neuropathologic studies reported neuronal alterations in several subcortical structures such as the olfactory tract/bulb, anterior olfactory nucleus, orbito-frontal cortex, hippocampus, and amygdala in the olfactory system [27]. Olfactory abnormalities have been reported in $\mathrm{AD}$, but anosmia appears to be common in DLB but not in pure $\mathrm{AD}$ [28]. Interestingly, a Lewy body variant of $\mathrm{AD}$ had an increased frequency of anosmia compared with "pure" $\mathrm{AD}$ [29]. Furthermore, olfactory impairment is more marked in patients with mild dementia with Lewy bodies than in those with mild $\mathrm{AD}$ [30]. In addition to reduced odor identification prior to the onset of $\mathrm{PD}$, olfactory dysfunction also has been frequently recognized in patients with ILBD [31]. Also, neuropathological olfactory bulb alpha-synclein has high specificity and sensitivity for Lewy body formation in confirmed cases of PD and DLB [32]. On the basis of pathological studies of a large number of autopsy cases, Braak et al. proposed a hypothesis as to the onset and advancement pattern of PD in that the disease developed from the medulla and olfactory bulb and extended to 
the pons and substantia nigra (SN) [33]. In the HonoluluAsia Aging Study on Japanese Americans, 2,267 males without PD and dementia at the time of olfaction testing were followed up and significantly more subjects who developed olfactory dysfunction in the first 4-year follow-up period also developed PD [34]. Haehner's study was to clinically follow up patients with idiopathic hyposmia to determine the percentage of patients who developed idiopathic PD after a 4-year interval [35]. In Ponsen's prospective study involving first-degree relatives of PD patients, a low score on three olfactory processing tasks was associated with an increased risk of developing PD within 5 years [36]. The point of view that reduced odor identification is manifested at the very beginning of the development of PD has been supported. These reports indicate that the rhinencephalon may be an area of selective vulnerability for $\alpha$-synuclein accumulation [37] and iRBD that develops in middle age or older progresses mostly to Lewy body diseases among synucleinopathies such as PD and DLB, which have some pathological features in common.

4.3. RBD and Relevance to Lewy Body Pathology. Men over the age of 50 years who have $\mathrm{iRBD}$ are at very high risk for future PD or DLB several years after the onset of RBD $[38,39]$. Neuropathologic studies at autopsy of cases that had been diagnosed with RBD while alive showed that every case had Lewy bodies [6]. For example, cognitive abnormalities appeared 15 years after the onset of RBD and probable DLB was diagnosed, and eventually the presence of Lewy bodies was confirmed pathologically [40]. In PD, approximately $60 \%$ of the nigrostriatal neurons of the substantia nigra are degenerated before patients fulfill the clinical criteria of PD [41]. In some cases of PD, the patient appears to develop cortical disease before the motor sign of "stage 3" disease, whereas, iRBD patients with ILBD could be diagnosed after long-standing disease with no evidence of motor or cognitive abnormalities $[3,4]$. If progression of syncleinopathies is not universal, it is essential to understand the reason. Since a variety of symptoms of PD and disorders resembling PD have been elucidated, Langston [42] proposed a "Parkinson's complex" because parkinsonism would represent only the tip of the iceberg as typically viewed by both clinicians and researchers. However, when the disease process is measured by neuronal degeneration, the presence of Lewy bodies and neuritic pathology are widespread in the central and peripheral nervous systems. From this point of view, iRBD can be positioned as an earlier preclinical stage of PD or DLB, or a variant of Lewy body-related $\alpha$-synucleinopathies. To gain such an understanding, it is necessary to extract a group with abnormalities in a combination of markers from among iRBD patients and provide follow up, considering the possibility that some patients in that group may develop neurodegenerative disease. These steps may help elucidate the possibility that iRBD is the spectrum of manifestations or a subtype of Lewy body-related $\alpha$-synucleinopathies.

In conclusion, reduced cardiac ${ }^{123}$ I-MIBG uptake may be more closely associated with iRBD than olfactory impairment. Moreover, odor identification impairment or cardiac sympathetic function assessed by cardiac ${ }^{123}$ I-MIBG uptake and the nigrostriatal dopaminergic function would occur and progress independently in iRBD patients or PD patients.

Limitations of this study include the small number of OSAS patients that comprise the control group. Untreated sleep apnea syndrome may be associated with physical movements during sleep [1], and, therefore, it is important to first differentiate between those with iRBD and OSAS with abnormal sleep behavior. A second limitation is that more than $80 \%$ of patients presenting at sleep centers with iRBD are men $[1,3,39]$ so that the male-to-female ratio was not matched in the $\mathrm{RBBD}, \mathrm{PD}$, and control groups.

\section{Conflict of Interests}

The authors report no financial conflict of interest. This work is not an industry supported study.

\section{Acknowledgments}

The authors are indebted to their colleagues with whom they work on the RBD, namely, Y. Inoue (Japan Somnology Center, Neuropsychiatry Research Institute and Department of Somnology, Tokyo Medical University). The authors thank T. Hashimoto (Department of Radiology, Dokkyo Medical University School of Medicine) for his helpful comments.

\section{References}

[1] C. H. Schenck and M. W. Mahowald, "REM sleep behavior disorder: clinical, developmental, and neuroscience perspectives 16 years after its formal identification in sleep," Sleep, vol. 25, no. 2, pp. 120-138, 2002.

[2] American Academy of Sleep Medicine, "Internal classification of sleep disorders," in Diagnosis and Cording Manual, pp. 148152, American Academy of Sleep Medicine, Westchester, Ill, USA, 2nd edition, 2005.

[3] J. F. Gagnon, R. B. Postuma, S. Mazza, J. Doyon, and J. Montplaisir, "Rapid-eye-movement sleep behaviour disorder and neurodegenerative diseases," Lancet Neurology, vol. 5, no. 5, pp. 424-432, 2006.

[4] T. Miyamoto, M. Miyamoto, Y. Inoue, Y. Usui, K. Suzuki, and K. Hirata, "Reduced cardiac ${ }^{123}$ I-MIBG scintigraphy in idiopathic REM sleep behavior disorder," Neurology, vol. 67, no. 12, pp. 2236-2238, 2006.

[5] T. Miyamoto, M. Miyamoto, K. Suzuki, M. Nishibayashi, M. Iwanami, and K. Hirata, "I-MIBG cardiac scintigraphy provides clues to the underlying neurodegenerative disorder in idiopathic REM sleep behavior disorder," Sleep, vol. 31, no. 5, pp. 717-723, 2008.

[6] B. F. Boeve, M. H. Silber, C. B. Saper et al., "Pathophysiology of REM sleep behaviour disorder and relevance to neurodegenerative disease," Brain, vol. 130, no. 11, pp. 2770-2788, 2007.

[7] P. H. Lee, S. H. Yeo, H. J. Kim, and H. Y. Youm, "Correlation between cardiac ${ }^{123}$ I-MIBG and odor identification in patients with Parkinson's disease and multiple system atrophy," Movement Disorders, vol. 21, no. 11, pp. 1975-1977, 2006.

[8] D. S. Goldstein and L. Sewell, "Olfactory dysfunction in pure autonomic failure: implications for the pathogenesis of Lewy body diseases," Parkinsonism and Related Disorders, vol. 15, no. 7, pp. 516-520, 2009. 
[9] T. Miyamoto, M. Miyamoto, M. Iwanami, K. Suzuki, Y. Inoue, and K. Hirata, "Odor identification test as an indicator of idiopathic REM sleep behavior disorder," Movement Disorders, vol. 24, no. 2, pp. 268-273, 2009.

[10] A. J. Hughes, S. E. Daniel, L. Kilford, and A. J. Lees, "Accuracy of clinical diagnosis of idiopathic Parkinson's disease: a clinico-pathological study of 100 cases," Journal of Neurology Neurosurgery and Psychiatry, vol. 55, no. 3, pp. 181-184, 1992.

[11] M. M. Hoehn and M. D. Yahr, "Parkinsonism: onset, progression and mortality," Neurology, vol. 17, no. 5, pp. 427-442, 1967.

[12] S. Fahn and R. L. Elton, "Unified Parkinson's disease rating scale," in Recent Developments in Parkinson's Disease, S. Fahn, M. Goldstein, D. Marsden, and D. B. Calne, Eds., vol. 2, pp. 153-163, Macmillan, New Jersy, NJ, USA, 1987.

[13] J. Jankovic and A. S. Kapadia, "Functional decline in Parkinson disease," Archives of Neurology, vol. 58, no. 10, pp. 1611-1615, 2001.

[14] M. L. Bleecker, K. Bolla-Wilson, C. Kawas, and J. Agnew, "Agespecific norms for the Mini-Mental State Exam," Neurology, vol. 38 , no. 10 , pp. $1565-1568,1988$.

[15] A. Rechtschaffen and A. Kales, A Manual of Standardized Terminology, Techniques and Scoring Systems for Sleep Stages of Human Subjects, Brain Information Service / Brain Research Institute, UCLA, Los Angeles, Calif, USA, 1968.

[16] M. Iijima, M. Osawa, M. Momose et al., "Cardiac sympathetic degeneration correlates with olfactory function in Parkinson's disease," Movement Disorders, vol. 25, no. 9, pp. 1143-1149, 2010.

[17] F. Courbon, C. Brefel-Courbon, C. Thalamas et al., "Cardiac MIBG scintigraphy is a sensitive tool for detecting cardiac sympathetic denervation in Parkinson's disease," Movement Disorders, vol. 18, no. 8, pp. 890-897, 2003.

[18] K. Kashihara, M. Ohno, S. Kawada, and Y. Okumura, "Reduced cardiac uptake and enhanced washout of ${ }^{123}$ I-MIBG in pure autonomic failure occurs conjointly with Parkinson's disease and dementia with Lewy bodies," Journal of Nuclear Medicine, vol. 47, no. 7, pp. 1099-1101, 2006.

[19] M. Yoshita, J. Taki, K. Yokoyama et al., "Value of ${ }^{123}$ I-MIBG radioactivity in the differential diagnosis of DLB from AD," Neurology, vol. 66, no. 12, pp. 1850-1854, 2006.

[20] S. Orimo, A. Takahashi, T. Uchihara et al., "Degeneration of cardiac sympathetic nerve begins in the early disease process of Parkinson's disease," Brain Pathology, vol. 17, no. 1, pp. 2430, 2007.

[21] S. Orimo, T. Uchihara, A. Nakamura et al., "Axonal $\alpha$ synuclein aggregates herald centripetal degeneration of cardiac sympathetic nerve in Parkinson's disease," Brain, vol. 131, no. 3, pp. 642-650, 2008.

[22] H. Fujishiro, R. Frigerio, M. Burnett et al., "Cardiac sympathetic denervation correlates with clinical and pathologic stages of Parkinson's disease," Movement Disorders, vol. 23, no. 8, pp. 1085-1092, 2008.

[23] R. L. Doty, S. M. Bromley, and M. B. Stern, "Olfactory testing as an aid in the diagnosis of Parkinson's disease: development of optimal discrimination criteria," Neurodegeneration, vol. 4, no. 1, pp. 93-97, 1995.

[24] L. Silveira-Moriyama, C. Mathias, L. Mason, C. Best, N. P. Quinn, and A. J. Lees, "Hyposmia in pure autonomic failure," Neurology, vol. 72, no. 19, pp. 1677-1681, 2009.

[25] R. L. Doty, L. I. Golbe, D. A. McKeown, M. B. Stern, C. M. Lehrach, and D. Crawford, "Olfactory testing differentiates between progressive supranuclear palsy and idiopathic Parkinson's disease," Neurology, vol. 43, no. 5, pp. 962-965, 1993.
[26] G. K. Wenning, B. Shephard, C. Hawkes, A. Petruckevitch, A. Lees, and N. Quinn, "Olfactory function in atypical parkinsonian syndromes," Acta Neurologica Scandinavica, vol. 91, no. 4, pp. 247-250, 1995.

[27] P. S. Hubbard, M. M. Esiri, M. Reading, R. McShane, and Z. Nagy, "Alpha-synuclein pathology in the olfactory pathways of dementia patients," Journal of Anatomy, vol. 211, no. 1, pp. 117-124, 2007.

[28] R. H. McShane, Z. Nagy, M. M. Esiri et al., "Anosmia in dementia is associated with Lewy bodies rather than Alzheimer's pathology," Journal of Neurology Neurosurgery and Psychiatry, vol. 70, no. 6, pp. 739-743, 2001.

[29] J. M. Olichney, C. Murphy, C. R. Hofstetter et al., "Anosmia is very common in the Lewy body variant of Alzheimer's disease," Journal of Neurology, Neurosurgery and Psychiatry, vol. 76, no. 10, pp. 1342-1347, 2005.

[30] S. S. Williams, J. Williams, M. Combrinck, S. Christie, A. D. Smith, and R. McShane, "Olfactory impairment is more marked in patients with mild dementia with Lewy bodies than those with mild Alzheimer disease," Journal of Neurology, Neurosurgery and Psychiatry, vol. 80, no. 6, pp. 667-670, 2009.

[31] G. W. Ross, R. D. Abbott, H. Petrovitch et al., "Association of olfactory dysfunction with incidental Lewy bodies," Movement Disorders, vol. 21, no. 12, pp. 2062-2067, 2006.

[32] T. G. Beach, C. L. White, C. L. Hladik et al., "Olfactory bulb $\alpha$ synucleinopathy has high specificity and sensitivity for Lewy body disorders," Acta Neuropathologica, vol. 117, no. 2, pp. 169-174, 2009.

[33] H. Braak, K. Del Tredici, U. Rüb, R. A. I. De Vos, E. N. H. Jansen Steur, and E. Braak, "Staging of brain pathology related to sporadic Parkinson's disease," Neurobiology of Aging, vol. 24, no. 2, pp. 197-211, 2003.

[34] G. W. Ross, H. Petrovitch, R. D. Abbott et al., "Association of olfactory dysfunction with risk for future Parkinson's disease," Annals of Neurology, vol. 63, no. 2, pp. 167-173, 2008.

[35] A. Haehner, T. Hummel, C. Hummel, U. Sommer, S. Junghanns, and H. Reichmann, "Olfactory loss may be a first sign of idiopathic Parkinson's disease," Movement Disorders, vol. 22, no. 6, pp. 839-842, 2007.

[36] M. M. Ponsen, D. Stoffers, J. W. R. Twisk, E. CH. Wolters, and H. W. Berendse, "Hyposmia and executive dysfunction as predictors of future Parkinson's disease: a prospective study," Movement Disorders, vol. 24, no. 7, pp. 1060-1065, 2009.

[37] R. Sengoku, Y. Saito, M. Ikemura et al., "Incidence and extent of lewy body-related $\alpha$-synucleinopathy in aging human olfactory bulb," Journal of Neuropathology and Experimental Neurology, vol. 67, no. 11, pp. 1072-1083, 2008.

[38] C. H. Schenck, S. R. Bundlie, and M. W. Mahowald, "Delayed emergence of a parkinsonian disorder in $38 \%$ of 29 older, men initially diagnosed with idiopathic rapid eye movement sleep behavior disorder," Neurology, vol. 46, no. 2, pp. 388393, 1996.

[39] R. B. Postuma, J. F. Gagnon, M. Vendette, M. L. Fantini, J. Massicotte-Marquez, and J. Montplaisir, "Quantifying the risk of neurodegenerative disease in idiopathic REM sleep behavior disorder," Neurology, vol. 72, no. 15, pp. 1296-1300, 2009.

[40] R. S. Turner, C. J. D’Amato, R. D. Chervin, and M. Blaivas, "The pathology of REM sleep behavior disorder with comorbid Lewy body dementia," Neurology, vol. 55, no. 11, pp. 1730$1732,2000$.

[41] J. M. Fearnley and A. J. Lees, “Ageing and Parkinson's disease: substantia nigra regional selectivity," Brain, vol. 114, no. 5, pp. 2283-2301, 1991. 
[42] J. W. Langston, “The Parkinson's complex: parkinsonism is just the tip of the Iceberg," Annals of Neurology, vol. 59, no. 4, pp. 591-596, 2006. 


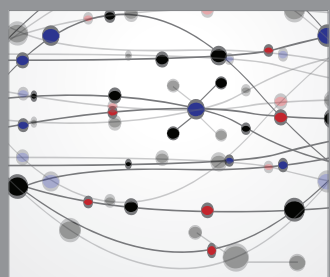

The Scientific World Journal
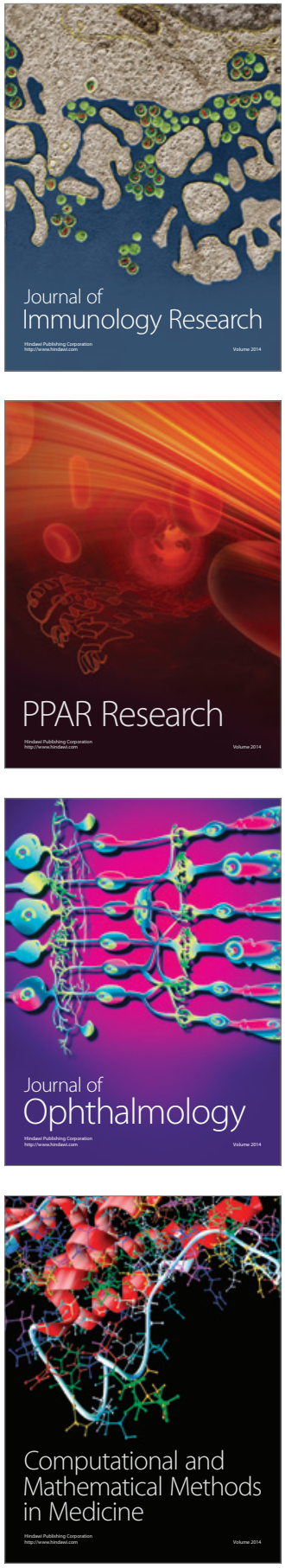

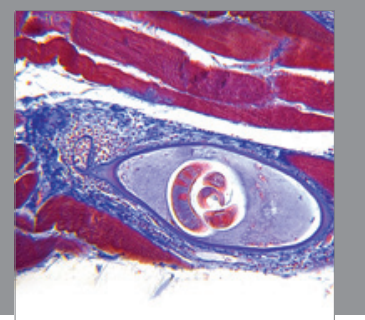

Gastroenterology

Research and Practice
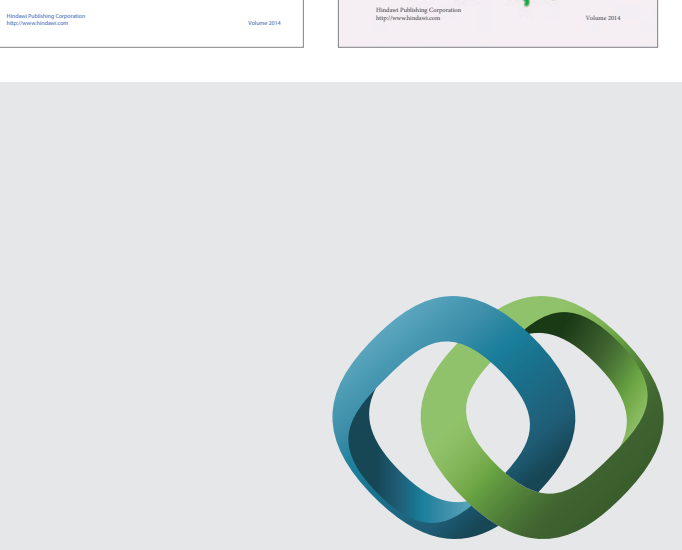

\section{Hindawi}

Submit your manuscripts at

http://www.hindawi.com
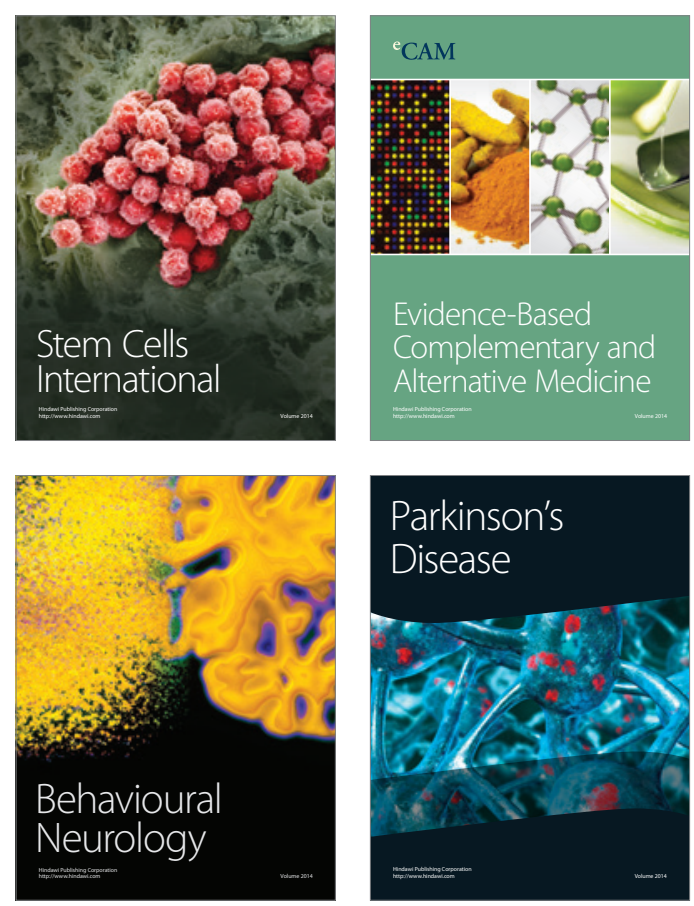

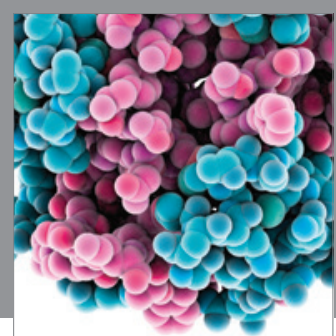

Journal of
Diabetes Research

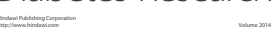

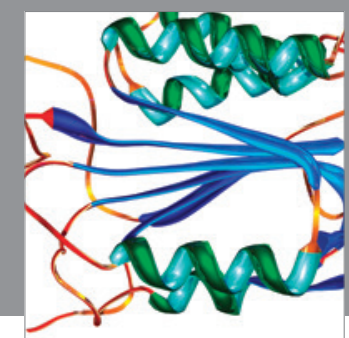

Disease Markers
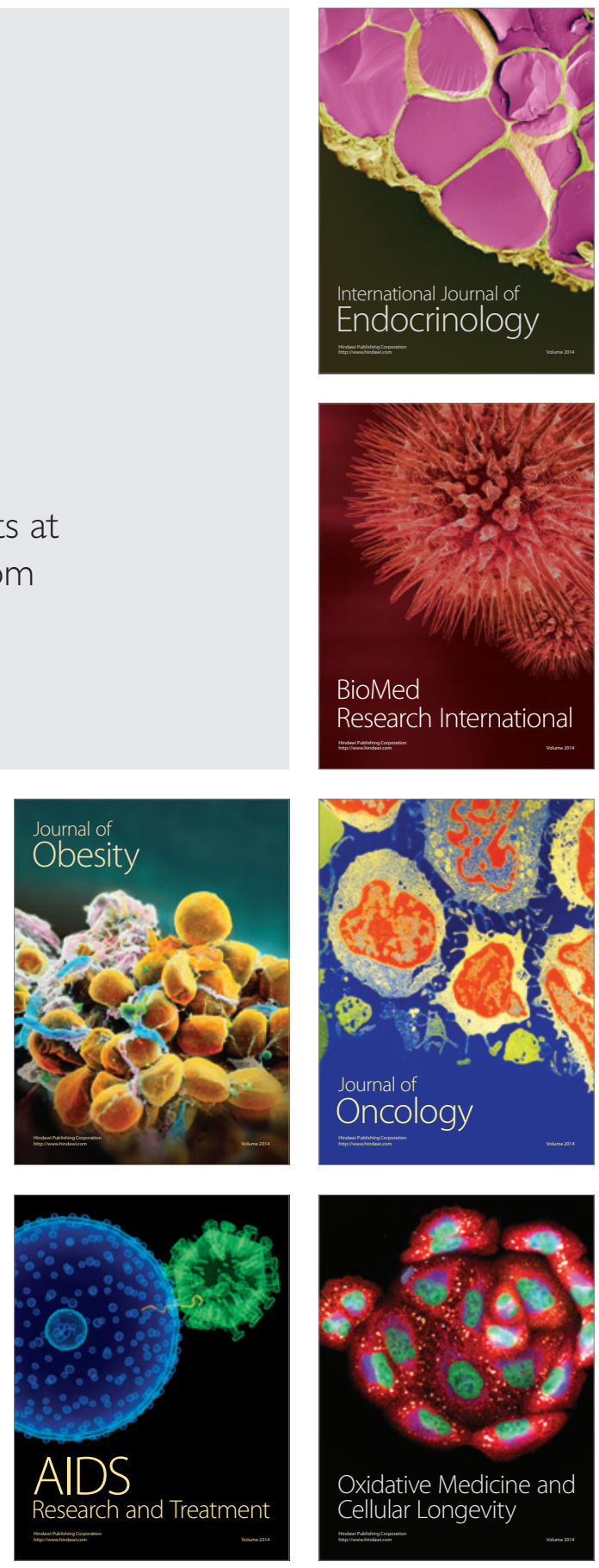